\title{
Transport of one-dimensional interacting Fermions through a barrier
}

\author{
Q. P. Li团 \\ Department of Physics and Applied Superconductivity Center \\ University of Wisconsin-Madison, 1150 University Avenue, Madison, Wisconsin 53706 \\ X. C. Xie \\ Department of Physics, Oklahoma State University \\ Stillwater, Oklahoma 74078-0444
}

(October 7, 2018)

\begin{abstract}
We study the transport properties of one-dimensional (1D) interacting Fermions through a barrier by numerically calculating the Kohn charge stiffness constant and the relative Drude weight. We find that the transport properties of the 1D Hubbard model are quite different from those of the 1D spinless Fermion model. For example, the presence of the attractive interaction between electrons in the 1D Hubbard model actually suppresses the DC conductance, while a small repulsive interaction enhances the DC conductance. These results show that the spin degree of freedom plays an important role in the transport properties of the 1D interacting Fermion systems.
\end{abstract}

PACS numbers: 71.30.+h, 75.10.Jm, 73.20.Dx

Typeset using REVTEX 
Recently, there has been increasing interest in one-dimensional (1D) electron systems. [1 8] On the one hand, the progress in ultrafine lithographic technology has made it possible to fabricate and experimentally study quantum wires in which only the lowest subband is occupied, approaching the true 1D limit; on the other hand, there is a renewed interest in the Luttinger liquid because of the suggestion that the high temperature superconductors might be non-Fermi-liquid-like. It is well known that even a weak interaction in a $1 \mathrm{D}$ Fermion system will drive the system to become a Luttinger liquid which exhibits quite different behavior from the Fermi liquid system. [9, 10] For example, Kane and Fisher have studied the transport properties in a 1D spinless Luttinger liquid with a barrier (or a weak link). They find that an attractive interaction makes the system a perfect conductor while a repulsive interaction makes it an insulator. [7]

In this paper we present some results of the transport properties of $1 \mathrm{D}$ interacting Fermion systems with a barrier. We study both the interacting spinless Fermion model and the 1D Hubbard model with a barrier by calculating the Kohn charge stiffness constant using the Lanczos algorithm. In particular, we investigate the effect of spin on the transport properties motivated by an interesting statement of Anderson who argued that the theory of the transport in 1D spinless Fermion may be irrelevant for a real electron system which has both charge and spin degrees of freedom. [11] We find that our result for the spinless Fermion model agrees with that of Kane and Fisher qualitatively. But our result of the 1D Hubbard model with a barrier is exact opposite of that of the spinless Fermion model. We find that in the case of the Hubbard model, the presence of the attractive interactions between electrons actually decreases the Kohn charge stiffness constant $\mathrm{D}_{c}$ as well as the relative Drude weight, in contrast to the 1D spinless Fermion case where the presence of attractive interactions increases $\mathrm{D}_{c}$ and the relative Drude weight as long as the attractive interaction is not large enough to cause a phase transition to a phase-separated insulating state. In the case of repulsive interaction, we find that a small repulsive interaction in the 1D Hubbard model with a weak link actually increases $\mathrm{D}_{c}$ and the relative Drude weight, again in contrast to the 1D spinless Fermion model where the repulsive interaction decreases 
$\mathrm{D}_{c}$.

We first study an interacting spinless Fermion model, so called Heisenberg-Ising model, on a $1 \mathrm{D}$ ring with a weak link

$$
H=-\sum_{i} t_{i}\left(c_{i}^{\dagger} c_{i+1}+\text { h.c. }\right)+V \sum_{i} n_{i} n_{i+1} .
$$

Here $t_{i}=t$ for $i \neq 1$ and $t_{1}=t^{\prime}$ representing the barrier (or weak link) site, and $\mathrm{V}$ is the nearest neighbor interaction. We assume the periodic boundary condition and thread the $1 \mathrm{D}$ ring with a flux $\Phi$ which is represented by a vector potential $\mathbf{A}=(\Phi / L) \hat{e}_{x}$, where $\mathrm{L}$ is the number of sites. We calculate numerically the ground state energy of the system $E(\Phi)$ as a function of flux, and consequently the Kohn charge stiffness constant

$$
D_{c}=\left.\frac{L}{2} \frac{d^{2} E(\Phi)}{d \Phi^{2}}\right|_{\Phi=0}
$$

$D_{c}$ is a measure of the electronic conductivity of the system, and it vanishes if the system is an insulator. Kohn first suggested that the charge stiffness constant can be used as a quantitative measure of the Mott metal-insulator transition. [12 15]

Physically, the charge stiffness constant $D_{c}$ is just the Drude weight in the real part of the optical conductivity $\sigma_{1}(\omega)$ in the long wavelength limit,

$$
\sigma_{1}(\omega)=2 \pi e^{2} D_{c} \delta(\omega)+\sigma_{1}^{r e g}(\omega)
$$

here we have set $\hbar=1$. Using second order perturbation theory, one finds, 16

$$
D_{c}=\frac{1}{2 L}<-T>-\frac{1}{L} \sum_{m \neq 0} \frac{|<0| j_{x}|m>|^{2}}{E_{m}-E_{0}}
$$

where $\langle-T\rangle$ is the expectation value of the kinetic energy in the ground state. $\sigma_{1}(\omega)$ satisfies the well-known f-sum rule,

$$
\int_{-\infty}^{\infty} \sigma_{1}(\omega) d \omega=\frac{\pi e^{2}}{L}<-T>
$$

We use the Lanczos algorithm to calculate the ground state energy. For a 14-site ring with 7 particles, the dimension of the Hilbert space is about 3400 and the Hamiltonian 
matrix has about 30,000 non-zero elements which can be handled easily on a workstation. We first consider a $1 \mathrm{D}$ ring with the lattice translational symmetry, i.e., $t^{\prime}=t$, in which most of the properties are known. This well-studied case provides us with a test example which we can check our computer program against and get some feelings about the finite size effect, etc. In Fig. 1a, we show the calculated ground state energy $E(\Phi)$ as a function of flux $\Phi$ for a 14-site ring with 7 and 6 particles, respectively. Obviously, $E(\Phi)$ is a periodic function of $\Phi$ with a period $\Phi_{0}=h c / e$. For the 7 particle system, the energy minimum is located at $\Phi=0$ as expected. This is true as long as the number of particles is an odd integer. But when there are even number of particles on a ring, such as in the 6-particle case, the minimum of $E(\Phi)$ is located at $\Phi=\Phi_{0} / 2$, signaling that the system prefers a spontaneous persistent current which would generate a flux of $\Phi_{0} / 2$. This is the well-known even-odd finite size effect. [17] Fig. 1b shows the calculated Kohn charge stiffness constant $D_{c}$ as a function of the nearest neighbor interaction $\mathrm{V}$ with $\mathrm{L}=14, \mathrm{~N}=7$, and $t^{\prime}=t$. One can see that on the repulsive interaction side, $D_{c}$ approaches zero quickly as the repulsive interaction increases, signaling a transition to the Mott-insulator in this half-filling case. On the attractive interaction side, $D_{c}$ also start to decrease, even faster than the repulsive case, at around $V=-2 t$. This is the transition to a phase-separated insulator state. On the repulsive interaction side, it is well known that $D_{c}$ and the effective mass have a discontinuous jump at $V=2 t$ in the thermodynamic limit. [16] As $V \rightarrow 2 t-0, \mathrm{D}_{c}$ approaches a nonzero value $1 / 4$, while $D_{c}=0$ as $V \rightarrow 2 t+0$. Our finite size calculation yields $D_{c} \simeq 0.285$ at $V=2 t$. At $\mathrm{V}=0, \mathrm{D}_{c}$ in the thermodynamic limit is $1 / \pi \simeq 0.3183$. Our calculation gives $D_{c} \simeq 0.3210$. The finite size calculation usually overestimates $\mathrm{D}_{c}$ because of the periodic boundary condition.

In Fig. 2a, we show $D_{c}$ as a function of $\mathrm{V}$ for a 5 -particle 16 -site system with $t^{\prime}=\mathrm{t}, 0.75 \mathrm{t}$, and $0.25 \mathrm{t}$, respectively. Fig. $2 \mathrm{~b}$ shows the relative Drude weight (RDW) which is defined as the ratio of the Drude weight $D_{c}$ and the total spectral weight $A_{t o t}=\int_{-\infty}^{\infty} \sigma_{1}(\omega) d \omega / 2 \pi e^{2}$ for the same system. The first thing we can see from Fig. 2 is that the presence of a weak link does not seem to affect the phase-separation transition much. The weak link actually makes the phase-separation-transition sharper in a finite size system. The transition is 
depicted better in the relative Drude weight plot (cf. Fig. 2b). It is obvious that any theory which assumes a uniform phase such as that of Kane and Fisher [7] is only applicable above the threshold $V_{c}=-2 t$. Below $V_{c}, D_{c}$ is zero and the system is an insulator in the thermodynamic limit. The second thing we observe from Fig. 2 is that the attractive interaction does enhance the conductivity, especially when $t^{\prime} / t$ is small, that is quite different from the result of 1D Hubbard model which we'll discuss later, while the repulsive interaction suppress the conductivity. This is in qualitative agreement with the analytical studies of Kane and Fisher [0], and Apel and Rice. [18] However, due to the finite size effect and the phase-separation transition at $V_{c}=-2 t$, presently we are not able to confirm Kane and Fisher's specific prediction [7] that any repulsive interaction will drive the system to insulating while any attractive interaction will make the system a perfect conductor. We find that $D_{c}$ and the relative Drude weight remains finite even for very large positive $\mathrm{V}$ in a finite system. The third point we learn from Fig. 2 is that when $t^{\prime}=t$, the Drude weight almost exhausts all the spectral weight $A_{t o t}$ as long as $\mathrm{V}$ is above the phase-separation threshold $V_{c}=-2 t$. The relative Drude weight drops quite rapidly once $\mathrm{V}$ is decreased below $V_{c}$. When $t^{\prime}<t$, the RDW is usually smaller than the $t^{\prime}=t$ case (cf. Fig. 2b). This is due to the pinning of the charge density wave by the weak link, which not only reduces $D_{c}$, but also reduces the RDW.

Next we consider the Hubbard model on a 1D ring with a weak link,

$$
H=-\sum_{i \sigma} t_{i}\left(c_{i \sigma}^{\dagger} c_{i+1 \sigma}+\text { h.c. }\right)+U \sum_{i} n_{i \uparrow} n_{i \downarrow} .
$$

Here again $t_{1}=t^{\prime}$ and $t_{i}=t$ for $i \neq 1$, and $\sigma=\uparrow, \downarrow$ is the spin index. In Fig. 3 we show the calculated $\mathrm{D}_{c}$ and the relative Drude weight as functions of the interaction $\mathrm{U}$ for $t^{\prime}=\mathrm{t}, 0.5 \mathrm{t}$, and $0.1 \mathrm{t}$, respectively. We find that the transport properties of the $1 \mathrm{D}$ hubbard model are quite different from those of the spinless Fermion model. In Hubbard model, the presence of the attractive interaction between electrons actually decreases $\mathrm{D}_{c}$ and RDW (cf. Fig. 3), in contrast to the spinless Fermion model (cf. Fig. 2 and Refs. [7, 18]). The result of the repulsive interaction is equally surprising. The presence of a small repulsive interaction 
$(U>0)$ increases $\mathrm{D}_{c}$ and RDW. So the maximum of $\mathrm{D}_{c}$ actually shifts from $\mathrm{U}=0$ for $t^{\prime}=t$ to some small positive $U_{0}\left(t^{\prime}\right)$ for $t^{\prime}<t$ (cf. Fig. 3). $U_{0}\left(t^{\prime}\right)$ increases as $t^{\prime}$ decreases. For $t^{\prime}=0.1 t, U_{0} \simeq t$. Since the only major difference between the Hubbard model and the spinless Fermion model is the spin, it is reasonable to suspect that the spin does play a very important role in the transport of $1 \mathrm{D}$ interacting Fermions, which is in agreement with Anderson's conclusion. 11]

The fact that in Hubbard model, $\mathrm{D}_{c}$ decreases as one increases the strength of the attractive interaction can be understood from the following physical picture. In the attractive interaction region, electrons form pairs which can be treated as hard-core bosons, [19], and these bosons can hop via virtual ionization. A straight forward second-order perturbation calculation yields that the pair hopping amplitude is $2 t^{2} /|U|$ and the nearest neighbor repulsion between the bosons is also $2 t^{2} /|U|$ in the $t^{\prime}=t$ case. Physically, this is easy to understand because the virtual ionization to a nearby site lowers the energy, but it is only possible if the nearby site is empty. [20]

Comparing our results with that of Ref. 7 and 8, we find that although our results in the spinless case is in qualitative agreement with their results, there is significant discrepancy between our results and that of Ref. 7 and 8 in the spinful Fermion case. The Hubbard model we studied has $\mathrm{SU}(2)$ symmetry, so $g_{\sigma}$ is fixed at 2 (we use the same notation $g_{\sigma}$ as in Ref. 7). This means, according to Ref. 7 and 8, that the spin part should behave like the noninteracting case while the charge part should act like the spinless Fermion. This is in disagreement with our finding that the presence of a small repulsive interaction in Hubbard model with a week link actually increases DC conductance which is quite different from the results of spinless Fermions. One possible explanation of our results is that the presence of repulsive interaction increases the antiferromagnetic correlation which may enhances the transport across the weak link. Some of these physics is apparently missing in Ref. 7 and 8. For example, Ref. 8 starts from a model which only has interaction $g_{2}$ and completely neglects the $2 k_{F}$ component $g_{1}$. Here $g_{1}$ and $g_{2}$ are standard "g-ology" notations as in Ref. 9 . 
Finally we mention that although all of the results shown above are for $t^{\prime} \leq t$, we have also studied the case of $t^{\prime}>t$. In this case, we find that $\mathrm{D}_{c}$ decreases as $t^{\prime}$ is increased from $\mathrm{t}$, in agreement with the picture that the $t^{\prime} \neq t \operatorname{link}$ (either $t^{\prime}<t$ or $t^{\prime}>t$ ) serves as a pinning center of the charge density wave in the repulsive interaction region.

In conclusion, we have studied the transport properties of both the 1D spinless Fermion model and the Hubbard model with a weak link by calculating the Kohn charge stiffness constant $\mathrm{D}_{c}$ and the relative Drude weight using Lanczos algorithm. We find that the presence of the attractive interaction between electrons in 1D Hubbard model actually suppresses the DC conductivity, which is in contrast to the 1D spinless Fermion model where the attractive interaction enhances the DC conductivity as long as the attractive is not large enough to cause a phase transition to a phase-separated insulating state. In the repulsive interaction region, we find that a small repulsive interaction in 1D Hubbard model actually increases $\mathrm{D}_{c}$ and the relative Drude weight, again in contrast to the 1D spinless Fermion model where the repulsive interaction decreases $\mathrm{D}_{c}$. These results show that the spin degree of freedom plays an important role in the transport properties of the 1D interacting Fermion systems.

\section{ACKNOWLEDGMENTS}

It is a pleasure to thank S. Das Sarma, Steve Girvin, Song He, Ben Hu, Robert Joynt, G. Kotliar, Qian Niu, C. Stafford, Shuan Tang, and Nandini Trivedi for valuable conversations. QPL would like to thank the hospitality of the Aspen Center for Physics where part of the

work was done. This work was supported in part by the NSF through Grant No. DMR 9214739, and by the Electric Power Research Institute. We also acknowledge support from San Diego Supercomputer Center. 


\section{REFERENCES}

* Present address: Department of Physics, University of Maryland, College Park, Maryland 20742.

[1] W. Hansen, M. Horst, J. P. Kotthaus, U. Merkt, Ch. Sikorski, and K. Ploog, Phys. Rev. Lett. 58, 2586 (1987).

[2] B. J. Van Wees et al., Phys. Rev. Lett. 60, 848 (1988); Phys. Rev. B 38, 3625 (1988).

[3] J. H. F. Scott-Thomas, S. B. Field, M. A. Kastner, H. I. Smith, and D. A. Antoniadis, Phys. Rev. Lett. 62, 583 (1989).

[4] U. Meirav, M. A. Kastner, M. Heiblum, and S. J. Wind, Phys. Rev. B 40, 5871 (1989).

[5] A. R. Goni, A. Pinczuk, J. S. Weiner, J. M. Calleja, B. S. Dennis, L. N. Pfeiffer, and K. W. West, Phys. Rev. Lett. 67, 3298 (1991).

[6] Q. P. Li and S. Das Sarma, Phys. Rev. B 43, 11768 (1991); ibid 44, 6277 (1991); Q. P. Li, S. Das Sarma, and R. Joynt, Phys. Rev. B 45, 13713 (1992).

[7] C. L. Kane and M. P. A. Fisher, Phys. Rev. Lett. 68, 1220 (1992); Phys. Rev. B 46, 15233 (1992).

[8] A. Furusaki and N. Nagaosa, Phys. Rev. B 47, 4631 (1993).

[9] J. Solyom, Adv. Phys. 28, 201 (1970); V. J. Emery, in Highly Conducting OneDimensional Solids, edited by J. T. Devreese, R. P. Evrard, and V. E. van Doren (Plenum, New York, 1979).

[10] F. D. M. Haldane, J. Phys. C 14, 2585 (1981).

[11] P. W. Anderson, in Princeton RVB book, Chap. 5 (unpublished).

[12] W. Kohn, Phys. Rev. 133, A171 (1964).

[13] A. J. Millis and S. N. Coppersmith, Phys. Rev. B 43, 13770 (1991). 
[14] D. Poilblanc and E. Dagotto, Phys. Rev. B 44, 466 (1991).

[15] Q. P. Li and R. Joynt, Phys. Rev. B 47, 3979 (1993).

[16] B. S. Shastry and B. Sutherland, Phys. Rev. Lett. 65, 243 (1990).

[17] B. Doucot and X. G. Wen, Phys. Rev. B 40, 2719 (1989).

[18] W. Apel and T. M. Rice, Phys. Rev. B 26, 7063 (1982).

[19] In 1D Hubbard model with an attractive interaction, a pair of electrons always form a bound state as long as the attractive interaction is nonzero. See Ref. [15].

[20] P. Nozieres and S. Schmitt-Rink, J. Low Temp. Phys. 59, 195 (1985). 


\section{FIGURES}

FIG. 1. (a). Shows the calculated ground state energy $E(\Phi)$ of the Heisenberg-Ising model as a function of the flux $\Phi$ for $\mathrm{N}=7$ (solid) and 6 (dashed), respectively. Here $\mathrm{L}=14, \mathrm{~V}=2 \mathrm{t}$, and $t^{\prime}=t$. The unit of $E$ is t. (b). Shows the calculated $D_{c}$ as a function of interaction $\mathrm{V}$ for the $\mathrm{N}=7$ system.

FIG. 2. Shows the calculated (a) $D_{c}$ and (b) the relative Drude weight as a function of interaction $\mathrm{V}$ of a 5 -spinless-Fermion system on a 16 -site $1 \mathrm{D}$ ring with $t^{\prime}=\mathrm{t}$ (solid), $0.75 \mathrm{t}$ (dashed), and $0.25 \mathrm{t}$ (dot-dashed), respectively.

FIG. 3. Shows the calculated (a) $D_{c}$ and (b) the relative Drude weight of a 1D Hubbard model as a function of interaction $\mathrm{U}$ of a 6 -Fermion system on a 8-site ring with $t^{\prime}=\mathrm{t}$ (solid), $0.5 \mathrm{t}$ (dashed), and $0.1 \mathrm{t}$ (dot), respectively. The total spin $S_{z}=0$. 